\title{
Frontières
}

\section{Rieff, David, Mort d'une inconsolée, Les derniers jours de \\ Susan Sontag, Paris, Climat, 2008, 182 p.}

\section{Hélène Houde}

Volume 21, numéro 1, automne 2008

Prévenir le suicide

URI : https://id.erudit.org/iderudit/037891ar

DOI : https://doi.org/10.7202/037891ar

Aller au sommaire du numéro

\section{Éditeur(s)}

Université du Québec à Montréal

ISSN

1180-3479 (imprimé)

1916-0976 (numérique)

Découvrir la revue

Citer ce compte rendu

Houde, H. (2008). Compte rendu de [Rieff, David, Mort d'une inconsolée, Les derniers jours de Susan Sontag, Paris, Climat, 2008, 182 p.] Frontières, 21(1),

145-146. https://doi.org/10.7202/037891ar d'utilisation que vous pouvez consulter en ligne.

https://apropos.erudit.org/fr/usagers/politique-dutilisation/ 
tu lui fais remarquer que tu as oublié ta raquette à la maison. Tu retournes la chercher, [...] Ta femme [...] elle est restée dehors, il fait beau, elle profite du soleil. Quelques instants plus tard, elle entend la décharge d'une arme à feu. Tu t'es tiré une balle dans la tête avec le fusil que tu avais soigneusement préparé.

Sur un ton froid et distancié, le narrateur va brosser le portrait de l'ami suicidé, qui est à la fois présent au monde et détaché de lui. Levé le présente comme un être stoïque, délicat et sensible, ne voulant pas décevoir, parlant peu, regardant et écoutant beaucoup les autres. S'il lui était facile de rencontrer des gens nouveaux en tête-à-tête, il lui était, par contre, difficile d'en rencontrer en groupe. II parlait peu, mais avec précision, et avec passion lorsque son interlocuteur lui était familier. Pas misanthrope, mais pas mondain non plus, il savait dialoguer avec n'importe qui, et préférait le mode de l'interrogation à celui de l'affirmation.

Ailleurs, le narrateur relève le côté solitaire de l'ami suicidé: il aimait se promener seul dans des villes inconnues, naviguer en solitaire, s'enfermer dans sa chambre. Cependant, d'autres aspects plus sociables tempèrent ce côté solitaire, puisqu'il aimait aussi jouer de la batterie et le faisait avec virtuosité, et qu'il nageait, courait, montait à cheval, jouait avec ses amis, au tennis, au squash et au ping-pong.

Le narrateur précise que l'ami suicidé avait une famille, des amis, une maison, qu'il ne manquait pas d'argent et n'était ni solitaire, ni pauvre, ni alcoolique. II réussissait le peu qu'il entreprenait car il choisissait de faire «peu mais bien, ou rien plutôt que mal ». Pourtant, il ne s'identifiait pas aux gens heureux, mais se projetait dans ceux qui avaient tout raté, ou rien réussi, comme les clochards.

Étant donné que le narrateur ne fait que décrire sans jamais analyser, le mystère du suicide de l'ami reste entier. En effet, le récit n'a pas pour but d'expliquer le suicide: "Expliquer ton suicide? Personne ne s'y est risqué. " II se contente de donner naissance à toutes sortes $d$ 'hypothèses sans fournir de réponses, bien qu'il donne quelques indices sur la présence d'un mal-être, au quotidien, qui pèse et étouffe «[...] impression de ne pas être soi en se regardant dans la glace, impression d'être ailleurs en étant quelque part, sensations irréelles [...] Jeune encore, ton désarroi était inconsolable parce que tu le jugeais infondé ».
Par contre, le narrateur parle abondamment de la mort choisie, préméditée, familière, qui n'est pas une négation de la vie, mais une affirmation d'un "goût pour l'inconnu en pariant que si, de l'autre côté, quelque chose existait, ce serait mieux qu'ici». On comprend qu'il s'agit d'une quête de bonheur qui va tellement loin qu'elle va audelà de la vie et prend le risque de n'y rien trouver. Notons que le narrateur nous met en garde contre la tentation d'expliquer toute une vie par le suicide, il nous dit que celui-ci ne doit pas devenir «le prisme essentiel, unique, à travers lequel tous les autres événements qui l'ont précédé doivent se lire, voire se comprendre."

Récit très troublant car au fur et à mesure qu'il avance, il nous semble qu'en fait le tu s'est substitué au je et que plus le narrateur nous parle de son ami, plus il nous parle de lui-même, de ce qu'il pense de la vie, de la mort et de sa propre mort volontaire à venir. En réalité, par le truchement de ce récit, il vit, par anticipation, sa mort et ses conséquences sur ses proches. Les similitudes avec l'ami suicidé étant trop nombreuses, il semblerait bien que Suicide nous mette en présence du double d'Édouard Levé. Soulignons notamment que les deux personnages ont tellement apprivoisé l'idée de leur mort qu'ils n'ont pas craint de franchir le pas qui les en séparait. De plus, tous deux n'ont jamais fait de tentatives de suicide ratées et ont, par contre, planifié leur mort jusque dans les moindres détails, sans rien laisser au hasard; Édouard Levé est allé jusqu'à laisser, bien en évidence, des lettres adressées à sa femme et à ses amis.

En fait, il ne faudrait pas s'étonner de ce dédoublement puisque le thème et le trouble de l'identité faisaient déjà partie des préoccupations de Levé, comme en témoigne son œuvre photographique: en 1999, sa première série, Homonyme, est une suite de clichés d'hommes ayant le même nom que des auteurs célèbres comme Henri Michaux, Eugène Delacroix ou Yves Klein. Un autre travail photographique, qu'il a réalisé sur des villes homonymes américaines: Bagdad, Amsterdam, Rome, témoigne aussi d'une préoccupation semblable.

En écrivant Suicide, Levé nous annonce qu'il a fait le choix de faire venir sa mort et de l'accueillir plutôt que de l'attendre, d'affirmer son libre arbitre en fixant l'échéance de l'inéluctable. Cette mort volontaire ne l'a pas empêché d'éprouver des regrets pour la tristesse qu'il allait causer à ses proches. D'ailleurs, Levé leur laissera des lettres qui leur éviteront des interrogations sans réponses et des interprétations erronées. Remarquons que son souci de déculpabiliser les proches est déjà présent dans Suicide quand il précise: «Personne d'autre que toi ne pouvait te donner plus de goût pour la vie que pour la mort [...]".

Avec Suicide, un livre vraiment pas comme les autres, Levé s'interroge sur l'existence, prolonge cette vie qu'il a voulu abréger, puisque grâce à l'œuvre littéraire il ouvre la porte à toutes sortes $d$ 'hypothèses concernant sa vie et sa mort, se survit d'une certaine manière et "donne vie à la mort". L'auteur nous offre un récit beau, puissant, sans fioritures, d'une grande sobriété, qui dégage une angoisse métaphysique profonde. Ses phrases directes, nettes et incisives ne peuvent laisser le lecteur indifférent; le récit se termine par une suite de tercets, dont le dernier a un accent prémonitoire:

Le bonheur me précède,

La tristesse me suit,

La mort m'attend.

On ne peut que regretter la disparition prématurée d'une personnalité attachante et d'un être talentueux qui ne sera pas oublié de si tôt.

\section{Shahira El Moutei-Khalil}

\section{RIEFF, David \\ Mort d'une inconsolée Les derniers jours de Susan Sontag}

Paris, Climat, 2008, 182 p.

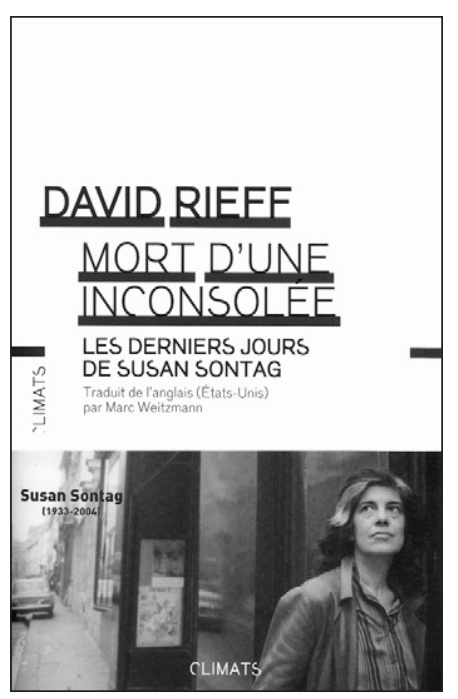

Une mère mourante, un fils présent. Elle, Susan Sontag, connue mondialement pour ses écrits (entre autres pour La maladie comme métaphore, Le sida et ses métaphores). Lui, David Rieff, célèbre journaliste couvrant les grands conflits internationaux (au New York Times Magazine entre autres) et renommé éditeur américain de Marguerite Yourcenar, Elias Canetti, Philippe Roth. Derrière ces personnages publics, une femme et un homme affrontent la mort: deux personnalités et un fort lien d'affection. En mai dernier, j'ai assisté à une entrevue que David Rieff donnait à un média. J'ai vu se présenter devant nous un homme dans la cinquantaine, très grand, élancé, au regard franc et direct, à la démarche lente et prudente: un fils venu raconter la mort et l'agonie de sa mère, un homme venu dire l'impuissance ressentie et l'immensité de sa perte. Un parcours dur mais exceptionnel; du 28 mars 2004 au 28 décembre 2004, neuf mois!

Susan Sontag connaît la maladie qu'est le cancer: en 1975 (début de la quarantaine), elle subit une mammectomie radicale après la découverte d'un cancer du sein à un stade avancé; en 1998, elle subit une chirurgie et une chimiothérapie suite à la découverte d'un sarcome utérin. Quand au début 2004 on lui annonce que la biopsie de la moelle épinière révèle, sans le moindre doute, un syndrome myélodysplasique (cancer du sang), elle a 71 ans. Le médecin, sans prononcer le mot "mort», suggère d'attendre que la maladie se déclare (une leucémie myéloïde) pour agir. «En clair, articula-t-elle, avec une circonspection poignante [...], il n'y a rien à faire. Rien que je puisse faire » (p. 21). Et le silence s'installe entre la mère et le fils. Les mots qui normalement auraient dû les unir étaient devenus inutiles, manquants, inadéquats. Deux ans après la mort de sa mère, David Rieff nous raconte la vie, la maladie et la mort: celle de sa mère, la sienne, la leur.

Susan Sontag a toujours aimé la vie. À 16 ans elle écrivait dans son Journal (dont Rieff prépare la publication) qu'elle était « incapable de seulement imaginer qu'un jour [elle] ne serait plus en vie» (p. 21). "Elle ne pouvait imaginer autre chose qu'être » (p. 26). Elle croyait en son destin, en "sa bonne étoile». Ses espoirs en tant qu'écrivain étaient tels qu'elle voulait vivre jusqu'à cent ans, "incapable d'envisager la perspective de son extinction" (p. 30). Il était impensable qu'elle disparaisse: "Elle ne pouvait que se projeter dans l'étape suivante" (p. 160). Toujours plus de temps, jamais rassasiée, émerveillée, glou- 
tonne de tout ce qui se présentait à elle. Une femme d'espérance. Et pourtant! Son Journal met en lumière une enfance où elle se décrit comme «abandonnée et non aimée ». Rieff confie qu'« elle n'était pas d'un tempérament enthousiaste, au contraire; la plupart du temps il lui fallait se battre contre la dépression" (p. 145). Elle ne savait pas être heureuse dans l'instant présent. Elle allait jusqu'à dire que «sa vie privée était source de souffrance et de frustration » (p. 148). Dans ce monde d'espérance et de désespoir, il y avait avant tout chez Susan Sontag une "volonté de fer", une force psychologique, un besoin de combattre: ses armes à elles, celles qu'elle avait utilisées tout au long de sa vie. Quand à 71 ans, le médecin lui annonce qu'elle a un cancer, elle est prête à se battre à nouveau. Elle est fidèle à elle-même : "Quoi qu'il lui arrive, si contrariée, si défaite [...] elle finissait par se redresser, le regard fermement dirigé vers l'avenir - vers ce qui vient ensuite» (p. 154). Elle croyait en la raison, la science et les médecins. Ses relations avec le monde médical (à une exception près) ont été positives et constructives. Elle était prête à subir n'importe quel traitement expérimental qui permettrait une «commutation de peine - une grâce ", une rémission, du temps pour se remettre à écrire, "une nouvelle parcelle d'avenir», une prolongation. Pour ce faire, Susan Sontag avait « par-dessus tout [...] la volonté de ne pas fuir la douleur nécessaire, quelle qu'elle fût " (p. 48). Parallèlement à ce combat, s'insinuaient une profonde désespérance, une angoisse innommable, des "silences terrorisés» et la peur de la mort. Femme de mots et de verbe, ouverte et directe dans ses discussions, elle ne parlait pas de la mort avec son fils. Elle y pensait probablement constamment. Elle écrivit dans son Journal: "La mort est insupportable à moins de pouvoir dépasser le " Je " " (p. 170). Impossible pour elle. Non croyante, son athéisme a persisté jusqu'à la fin. Bref: "Elle est morte comme elle a vécu, c'est-à-dire irréconciliée avec la mort jusqu'au plus fort de la souffrance» (p. 23).

David Rieff, le fils, est là présent. Son rôle a été clairement défini par sa mère: "Elle exigeait de moi un refus inflexible d'envisager que cela [la mort] fut même possible» (p. 136). Trouver une «lueur d'espoir ", la réconforter sur les possibilités, les probabilités de survie (ou du moins de rémission), analyser positivement ses chances de guéri- son, telles étaient les responsabilités, les devoirs du fils vis-à-vis de sa mère - une situation extrêmement difficile à vivre, et pour elle et pour lui - « un impossible numéro d'équilibriste» (p. 136). Susan Sontag se définissait "comme quelqu'un dont l'appétit de vérité est absolu » (p. 112). Quel comportement adopter? Jusqu'où aller dans la recherche de l'information? Dire ou ne pas dire? Est-ce mensonge que ce "déni positif?" Était-ce le bon choix «ce calice empoisonné de l'espérance? » Deux ans après les événements, le fils ne sait toujours pas. Les pages où il confie sa culpabilité, son impuissance à comprendre, à soulager, sont tout particulièrement touchantes. II reconnaît ses relations tendues, difficiles avec sa mère, son "manque de tact et [sa] froideur", son « empressement à [se] faire une opinion et [s]'y tenir» (p. 131). II est toujours "dans l'océan » de la mort de sa mère. II y est encore immergé, baigné, englouti; «peu de pensées, beaucoup de regrets" (p. 180). La fin de Susan Sontag ne fut pas douce: "elle eut la mort que d'autres malades avaient eue avant elle [...] la mort qui rend tout savoir dénué de sens et toute volonté dénuée de sens, la mort qui rend inutile le talent des médecins " (p. 161). Une maladie sans pitié. Les derniers mots prononcés pour le fils, son unique enfant furent: "Je voulais te dire... ». Cet énoncé restera sans réponse. Pour toujours.

Ce livre est à retenir pour le style franc, la sobriété des mots et le témoignage d'une expérience d'accompagnement, de soutien en fin de vie. Parallèlement à ce récit rempli d'émotions, l'auteur soulève des questions plus générales liées au contexte de mort: Doit-on dire la vérité aux patients quant à leur état de santé et à l'application des traitements? Jusqu'où doit-on aller dans les traitements expérimentaux? "L'information, le savoir: sont-ils pouvoir ou cruauté? » Peut-on avoir trop d'informations? Trop peu d'informations? Que sous-entend une qualité de vie? Comment « encadrer l'espoir »? Le travail de deuil, un travail sans fondements? Beaucoup de questions, quelques ouvertures, une réelle réflexion.

Susan Sontag n'ayant pas laissé de directives quant à son inhumation, Rieff a décidé d'enterrer sa mère au cimetière Montparnasse de Paris. Il y passe quelquefois, mais y reste peu de temps, II ne croit pas qu'elle soit là. Mais son œuvre survit. À nous de l'y retrouver.

Hélène Houde

\section{Journal de chevet}

FEX, Gloria, Murielle Fex, Carmen Fex et Chantale Fex (2003).

Journal de chevet, Pour le cheminement de la personne / The Bedside Journal, To Assist the Person on the Journey, Gatineau, QuatroFex.

\section{Un historique du Journal de chevet par ses quatre créatrices}

L'idée de tenir un journal n'est pas nouvelle. Depuis longtemps, le journal intime est utilisé pour noter les pensées et les états d'âme. II a souvent un effet apaisant et réconfortant. Le journal de bord, quant à lui, sert à décrire les évènements du jour par ordre chronologique.

Le journal de chevet pour le cheminement de la personne se veut un outil de communication pour les personnes soignées à la maison, les personnes en résidence ou en établissement de santé et leurs familles.

Le journal de chevet a été conçu à la suite d'une série d'épisodes vécues avec notre mère. Le tout a débuté par un accident vasculaire cérébral (AVC) qui l'a laissée paralysée (hémi-

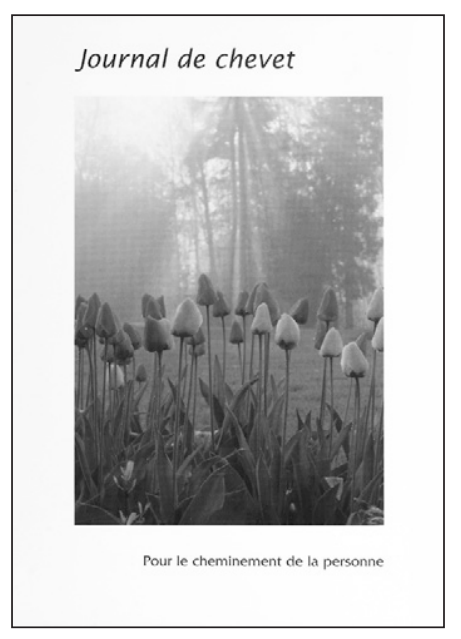

plégique). Nous avons dû alors trouver différents moyens de communication pour les membres de la famille et les divers intervenants.

Des petites notes sur un bout de papier au tableau d'affiche, de la planche de travail au cahier spiral, tous les moyens ont été utilisés lors de son séjour à l'hôpital, au centre de réadaptation et à son retour à la maison.

Le journal de chevet fut adopté comme outil de communication lors de I'hospitalisation de notre mère dans un centre de soins palliatifs. Issues d'une famille nombreuse, nous rendions visite à notre mère à tour de rôle. Comme plusieurs malades, notre mère avait des blancs de mémoire concernant les détails et événements de la journée. Donc, l'information se perdait et se déformait en cours de route.

Ce cahier d'écriture, laissé sur la table de chevet, nous gardait mutuellement au courant des événements et moments intimes vécus. De plus, nous avions ajouté à ce cahier les numéros de téléphone des personnes à contacter en cas d'urgence, les numéros également des amis à rejoindre pendant la journée et les événements à venir comme les fêtes des enfants. Nous notions également les achats d'effets personnels requis pour ses besoins et la personne mandatée pour les effectuer.

Les visiteurs du soir n'avaient pas la chance de rencontrer les médecins, les spécialistes, les techniciens et le personnel du jour pour connaître les changements et l'évolution de notre mère. Avec ce cahier de notes devenu journal de chevet, nous pouvions inscrire des remarques concernant l'état de notre mère et nous pouvions également laisser des notes au personnel de la santé qui changeait d'une journée à l'autre.

Ce journal peut être utilisé de maintes façons. Par exemple, la personne soignée peut rédiger elle-même son journal et le garder confidentiel ou une correspondance peut s'établir entre la personne, la parenté et les bénévoles qui pourraient lui témoigner de l'affection en rédigeant des pensées positives et des notes d'affection. Pour nous, ce journal de chevet est maintenant un souvenir précieux de la dernière phase de vie de notre mère et de tous ceux qui l'ont accompagnée tout au long du voyage.

Un an après le décès de notre mère, nous, les quatre sœurs Fex, avons décidé de partager cette expérience avec les intervenants du milieu de la santé et les personnes vivant une période difficile ou tragique.

Gloria Fex, Murielle Fex, Carmen Fex et Chantale Fex

Pour information, contacter:

Signature QuatroFex

Case postale 79075

Gatineau, Québec, J8Y 6V2

Courriel: gloriafex@videotron.ca 